\title{
Provider caseload volume and short-term outcomes following colorectal surgeries in New Brunswick: a provincial-level cohort study
}

\author{
Dan L. Crouse, PhD \\ Jonathan Boudreau, MSc \\ Philip S.J. Leonard, PhD \\ Keith Pawluk, MD \\ James T. McDonald, PhD
}

Accepted Jan. 7, 2020

\author{
Correspondence to: \\ D.L. Crouse \\ Health Effects Institute \\ 75 Federal St, Suite 1400 \\ Boston MA 02110 \\ dan.crouse@unb.ca
}

DOI: $10.1503 /$ cjs.012319

\begin{abstract}
Background: American studies have shown that higher provider and hospital volumes are associated with reduced risk of mortality following colorectal surgical interventions. Evidence from Canada is limited, and to our knowledge only a single study has considered outcomes other than death. We describe associations between provider surgical volume and all-cause mortality and postoperative complications following colorectal surgical interventions in New Brunswick.
\end{abstract}

Methods: We used hospital discharge abstracts linked to vital statistics, the provincial cancer registry and patient registry data. We considered all admissions for colorectal surgeries from 2007 through 2013. We used logistic regression to identify odds of dying and odds of complications (from any of anastomosis leak, unplanned colostomy, intra-abdominal sepsis or pneumonia) within 30 days of discharge from hospital according to provider volume (i.e., total interventions performed over the preceding 2 years) adjusted for personal, contextual, provider and hospital characteristics.

Results: Overall, 9170 interventions were performed by 125 providers across 18 hospitals. We found decreased odds of experiencing a complication following colorectal surgery per increment of 10 interventions performed per year (odds ratio 0.94, $95 \%$ confidence interval $0.91-0.96)$. We found no associations with mortality. Associations remained consistent across models restricted to cancer patients or to interventions performed by general surgeons and across models that also considered overall hospital volumes.

Conclusion: Our results suggest that increased caseloads are associated with reduced odds of complications, but not with all-cause mortality, following colorectal surgery in New Brunswick. We also found no evidence of volume having differential effects on outcomes from colon and rectal procedures.

Contexte : Des études américaines ont montré que le volume d'activité des chirurgiens et des hôpitaux est inversement proportionnel au risque de mortalité après la chirurgie colorectale. Les données pour le Canada sont limitées, et à notre connaissance, une seule étude a porté sur d'autres paramètres que le décès. Nous avons décrit les liens entre volume d'activité des chirurgiens et mortalité de toute cause/complications postopératoires après la chirurgie colorectale au Nouveau-Brunswick.

Méthodes : Nous avons utilisé les registres de congés des hôpitaux reliés aux données de la Statistique de l'état civil, du registre provincial du cancer et du registre des patients. Nous avons recensé toutes les admissions pour chirurgie colorectale de 2007 à 2013. Nous avons utilisé la régression logistique pour établir le risque de décès et le risque de complications (fuite anastomotique, colostomie non planifiée, infection intra-abdominale ou pneumonie) dans les 30 jours suivant le congé de l'hôpital par rapport au volume d'activité des chirurgiens (c.-à-d., interventions totales des 2 années précédentes) ajusté en fonction des caractéristiques individuelles et contextuelles, propres aux chirurgiens et aux hôpitaux.

Résultats : En tout, 125 chirurgiens ont effectué 9170 interventions dans 18 hôpitaux. Nous avons observé un risque moindre de complications après la chirurgie colorectale pour chaque palier de 10 interventions effectuées annuellement (risque relatif 0,94 , intervalle de confiance de $95 \%, 0,91-0,96$ ). Nous n'avons observé aucun lien avec la mortalité. Les liens sont demeurés constants, peu importe que les modèles soient restreints aux patients cancéreux ou aux interventions effectuées par des chirurgiens généraux et entre les modèles qui tenaient également compte du volume global d'activité des hôpitaux. 
Conclusion : Selon nos résultats, l'augmentation du volume d'activité est associée à un risque moindre de complications, mais n'a pas de lien avec la mortalité de toute cause après la chirurgie colorectale au Nouveau-Brunswick. Nous n'avons pas non plus constaté de lien entre le volume d'activité et l'issue différentielle de la chirurgie du côlon et du rectum.

A large body of literature has identified that for some surgical interventions, higher provider caseload volumes and higher hospital volumes are associated with improved patient outcomes. ${ }^{1-4}$ Interventions to improve outcomes for colorectal cancer have been well studied in the United States, with findings from many studies supporting the hypothesis that outcomes are sensitive to volumes at both the provider ${ }^{3}$ and hospi$\mathrm{tal}^{4}$ level. Inconsistencies in findings across studies are due in part to the wide variety of definitions of volume, ${ }^{5-9}$ and only a few have assessed whether provider volume is associated with outcomes while adjusting simultaneously for hospital volume. ${ }^{3,10}$ In their systematic review, Archampong and colleagues ${ }^{6}$ indicated that findings from American studies tended to differ from those of nonAmerican studies, suggesting that the health system context may influence the volume-outcome association for colorectal interventions and that individual countries should consider results from studies conducted with local data. For example, different countries have different general practices, training and organization of health care, and thus findings from one jurisdiction may not be immediately transferable to another.

In this context, evidence on the volume-outcome association for colorectal interventions in Canada is extremely limited. ${ }^{11-15}$ Three separate studies based on patients identified through the Ontario Cancer Registry reported no association between hospital volume and 30-day postoperative mortality following surgery among patients with rectal cancer $(n=1072),{ }^{11}$ patients with colon cancer $(n=$ $8398)^{12}$ and patients with colorectal cancer combined $(n=$ 18 898). ${ }^{13}$ A study based in British Columbia, however, identified positive associations between hospital volume and several quality indicators (i.e., operative and perioperative factors that influence either clinical, oncologic or quality-of-life outcomes) among roughly 2000 patients with rectal cancer. ${ }^{14}$ Additionally, a study of approximately 700 patients with rectal cancer in Edmonton found that colorectal surgical subspecialty training and increased volume of rectal cancer surgical interventions were associated with reduced risk of both local cancer recurrence and mortality. ${ }^{15}$

The vast majority of studies related to hospital or provider volume and interventions for colorectal cancer have considered only postoperative mortality (in both the shorter term [e.g., $30 \mathrm{~d}$ ] and the longer term [e.g., $5 \mathrm{yr}$ ]) as the outcome of interest. A few studies, however, have also examined indicators of quality of care and risk of complications, including readmission, reoperation ${ }^{16,17}$ and anastomotic leakage. ${ }^{18,19}$ Most of the previous Canadian studies on colorectal surgical interventions considered only hospital volumes (not provider volumes).

Given that the bulk of research on this topic has been conducted in the United States, with results that may not be generalizable to Canada or other jurisdictions, and that only a few Canadian studies ${ }^{14,15}$ have considered outcomes other than mortality, there is a need for more information on this topic from other jurisdictions. Here, we seek to describe associations between provider caseload volume and outcomes following colorectal surgical interventions, including mortality and postoperative complications, adjusted for hospital volume and other factors, among patients in New Brunswick (NB).

\section{Methods}

All analyses were conducted through the NB Institute for Research, Data and Training, which provides access to linked, pseudonymized, personal-level administrative health records. Record linkages are performed deterministically using patient obfuscated Medicare numbers. Our analyses are based on linked, provincial records from the provincial cancer registry (to identify patients diagnosed with cancer), the hospital discharge abstracts database (to identify details concerning diagnoses and interventions), the provider registry (to identify provider primary specialty and surgical experience), vital statistics (to identify patients that died) and the citizen database (to verify age and other patient characteristics).

Patient charts were reviewed by health record coders who indicated on the discharge abstracts up to 25 diagnoses and up to 20 interventions using the International Statistical Classification of Diseases and Related Health Problems, 10th Revision, enhanced Canadian version (ICD-10-CA) and the Canadian Classification of Health Interventions (CCI). The discharge abstracts indicate the timing of the diagnosis or intervention and the provider(s) (primary and otherwise) responsible for each intervention. The University of New Brunswick Research Ethics Board approved this study (file no. 2015-106).

\section{Cohort identification}

We identified all admissions for interventions related to selected colorectal surgical interventions in New Brunswick between Jan. 1, 2007, and Dec. 31, 2013, from the hospital discharge abstracts database (see Appendix 1, Supplemental Table S1, available at canjsurg.ca/012319-a1) for 
specific procedures and ICD-10-CA and CCI codes). We considered patient admissions from the 18 hospitals in the province where these procedures were performed. Along with information on diagnoses and interventions, the discharge abstracts include the date of the intervention, the hospital where the intervention took place, the surgical provider associated with each intervention, and the patient's age, sex, comorbidity level, urgency of admission and 6-digit residential postal code. Patient comorbidity was coded according to the Case Mix Groups+ methodology (CMG+) developed by the Canadian Institute for Health Information (www.cihi.ca/en/cmg). The CMG+ incorporates patient age, comorbidity level and other intervention-related details to aggregate acute care inpatients with similar clinical and resource-utilization characteristics. A higher score generally indicates a more complex case.

We used Statistics Canada's Postal Code Conversion File Plus $(\mathrm{PCCF}+)^{20}$ to geocode patients' residential postal codes at time of admission. In Canadian urban areas, the representative location of a 6-digit postal code corresponds typically to 1 side of a street in a given block or the centre of an apartment building, and it has positional accuracy within about 100-160 metres; in rural areas, there is greater positional uncertainty (i.e., typically accurate within about $1-5 \mathrm{~km}$ ). ${ }^{21,22}$ This locational information allowed us to identify the home community of each patient and thus determine the size of each patient's home community according to 2011 Statistics Canada Census data (i.e., cities with populations between 100000 and 499000 , small towns with populations between 10000 and 99999, and rural areas with populations of fewer than 10000 people). As an additional contextual variable, we calculated for each patient their neighbourhood income quintile (calculated as a household size-adjusted measure of household income on the basis of 2011 Census data at the dissemination area level). Dissemination areas are relatively small geographic units (generally composed of several city blocks in urban areas), cover all of Canada and have populations of approximately 400 to 700 people.

Among the patients identified above, we used the NB Provincial Cancer Registry to identify those who had been diagnosed with colorectal cancer in NB during the same period. Specifically, cancers were identified using the International Classification of Diseases for Oncology, Third Revision (ICD-O-3) topography codes: C18, C19, C20, and C26.0. These data also allowed us to characterize the stage of the cancer at the time of admission for surgery among those patients who did have colorectal cancer (i.e., stages 1-4; "no cancer" for those patients who did not have entries in the NB Provincial Cancer Registry; and "unstaged" for those individuals who did but for whom a diagnosis or a clear diagnosis level was not available at the time of the surgical procedure).

\section{Provider and hospital characteristics}

As noted above, the discharge abstracts list the surgical provider associated with each intervention, allowing linkage to the provincial provider registry, which includes the surgical histories for all providers in the province. Methods for defining physician experience and caseload volumes are highly heterogeneous in the existing literature ${ }^{7,8}$ in part because of differences in relative volumes between places. Here, we defined provider caseload volume as a continuous variable describing the annual mean number of procedure-specific interventions performed over the 2 years (i.e., $730 \mathrm{~d}$ ) preceding each new intervention. This approach allowed us to control for temporal changes in provider volume over the study period and has been applied elsewhere. ${ }^{23} \mathrm{We}$ also compiled information on each provider's primary specialization, sex and years since completing his or her basic medical training.

We also dichotomized the 18 hospitals into categories of relatively high and low volume in the context of admissions for colorectal surgical interventions after we conducted initial scans of the data and using our knowledge of the sizes and locations of the hospitals in the province. Specifically, we used the Canadian Institute for Health Information definitions, which consider factors such as number of inpatient cases and inpatient days, to code the 14 Community Small hospitals as relatively small and the 4 Community Large hospitals as relatively high. ${ }^{24}$ It is important to note that our data include records only for interventions performed in NB by NB-licensed providers.

\section{Outcomes}

We considered outcomes within 30 days of admission for colorectal surgical procedures. First, we considered mortality from all causes following surgery. Next, we considered incidence of any 1 of the following 4 complications: anastomosis leak, unplanned colostomy, intra-abdominal sepsis or pneumonia (see Appendix 1, Supplemental Table S2 for specific procedures and ICD$10-\mathrm{CA}$ and CCI codes) associated with these outcomes). We defined any colostomy that occurred within 30 days of discharge as unplanned. As such, we systematically excluded all colostomies that occurred as part of the original admission, which probably would have been planned. We selected these outcomes because they are relatively common, and initial scans of the data suggested variability in the rates of these outcomes across providers.

\section{Statistical analysis}

We used logistic regression to calculate the odds of complication following colorectal surgical interventions according to provider caseload volume controlling for several personal and contextual covariates. Our baseline 
models (model 1) were stratified by patient sex and age, in 5 -year groups. These models also included the calendar year of the intervention (to control for any potential secular trends in care practices), patient comorbidities, admission category (i.e., urgent v. nonurgent) and cancer stage. In the absence of personal-level socioeconomic information on patients, we also included size of home community and neighbourhood income quintile, as explained above, in our baseline models. Next (model 2), we included provider characteristics, including years of experience, sex and specialization (i.e., general surgery v. other). Note that in New Brunswick, the majority of colorectal surgical procedures are performed by general surgeons ( $75 \%$ of all interventions in this study). Here, our "other" category includes relatively small proportions of interventions performed by a mix of providers with specialties in internal medicine, gastroenterology, colorectal surgery or 1 of a few other surgical procedures. Third (model 3), for our fully-adjusted model, we included a categorical indicator of hospital volume.

To test the sensitivity of any associations observed across the full data set, we ran additional fully adjusted models in which first we restricted the sample to patients who had a cancer diagnosis (model 4). Next, we considered models in which we excluded all cases where the intervention was undertaken by a provider who had performed fewer than 2 of the listed interventions in the preceding 2-year period (model 5), as these providers may be visiting surgeons for whom we do not have complete surgical histories. Next, we considered models restricted to interventions performed by general surgeons (model 6). We considered these latter models because the majority of interventions were performed by general surgeons, whereas the "other" category was composed of only a few providers, some of whom had higher qualifications, and others who may have had unrelated additional specializations. Owing to the institutional confidentiality protection policies of the NB Institute for Research, Data and Training, we are unable to release or present results on any of these smaller subgroups individually. Finally, we considered models that included an interaction term between provider volume and an indicator of whether the procedure was related to the colon or the rectum. Here we sought to identify whether provider volumes had differential effects on the likelihood of adverse outcomes between interventions on the colon and rectum. All odds ratios were computed per increment of 10 interventions per year, annualized over the preceding 2 years.

\section{Results}

In total, 8475 patients were admitted for a colorectal surgical procedure during the study period. Overall, 9170 interventions were performed by 125 different providers across the 18 provincial hospitals. Of these patients,
$51.0 \%$ were male, their mean age was 63 years and $23.0 \%$ had been diagnosed with colorectal cancer. On average, 510 interventions were performed annually in each of the 4 higher volume hospitals, and 160 were performed in each of the 14 lower volume hospitals. Among all providers, the median annual volume was 17.9 interventions, and the vast majority $(93.6 \%)$ of all interventions were performed by a provider who had performed between 1 and 24 interventions in the previous year.

We present in Table 1 the distributions of several accepted and potential risk factors for adverse outcomes following surgical interventions among patients admitted to the smaller and larger volume hospitals. Overall, approximately two-thirds of all interventions were performed in the higher volume hospitals, and the majority (71.4\%) of all admissions were nonemergent. For confidentiality reasons, we do not report results according to provider sex because of the small number of female providers $(n=20)$.

In total, 120 patients died within 30 days of their procedure, and 1000 complications (375 anastomosis leaks, 495 unplanned colostomies, 275 cases of intraabdominal sepsis and 100 cases of pneumonia) were observed among 955 patients. In our fully adjusted model, patients admitted for urgent interventions had increased odds of dying within 30 days of surgery (odds ratio [OR] 3.18, 95\% confidence interval [CI 1.84-5.49) but no increased odds of complications compared with patients admitted for nonurgent interventions. Patients with multiple comorbidities had elevated odds of dying and of experiencing complications compared with those with no comorbidities. Our results suggested that patients with more advanced stages of cancer had increased odds of both mortality and complications, but none of these associations were statistically significant.

Having an intervention performed by a provider with specialization other than general surgery was associated with reduced odds of complication (OR $0.33,95 \%$ CI $0.26-0.42$ ) but not with reduced odds of dying (OR 0.95 , 95\% CI 0.59-1.54). Unexpectedly, we found that patients whose provider had more than 10 years' experience had higher odds of complication than those whose provider had fewer than 10 years' experience. Patients admitted to lower volume hospitals had lower odds of complication than those admitted to higher volume hospitals (OR 0.59, $95 \%$ CI $0.48-0.72$ ). None of the other risk factors included in our models were significantly associated with either all-cause mortality or odds of complication.

In Figure 1 we present associations between 30-day postoperative mortality following colorectal interventions and provider caseload volumes across our 6 different modelling scenarios. We found some suggestion of decreased odds associated with increased volumes, but no significant associations.

In Figure 2 we present the associations between the odds of complication and provider caseload volumes, 


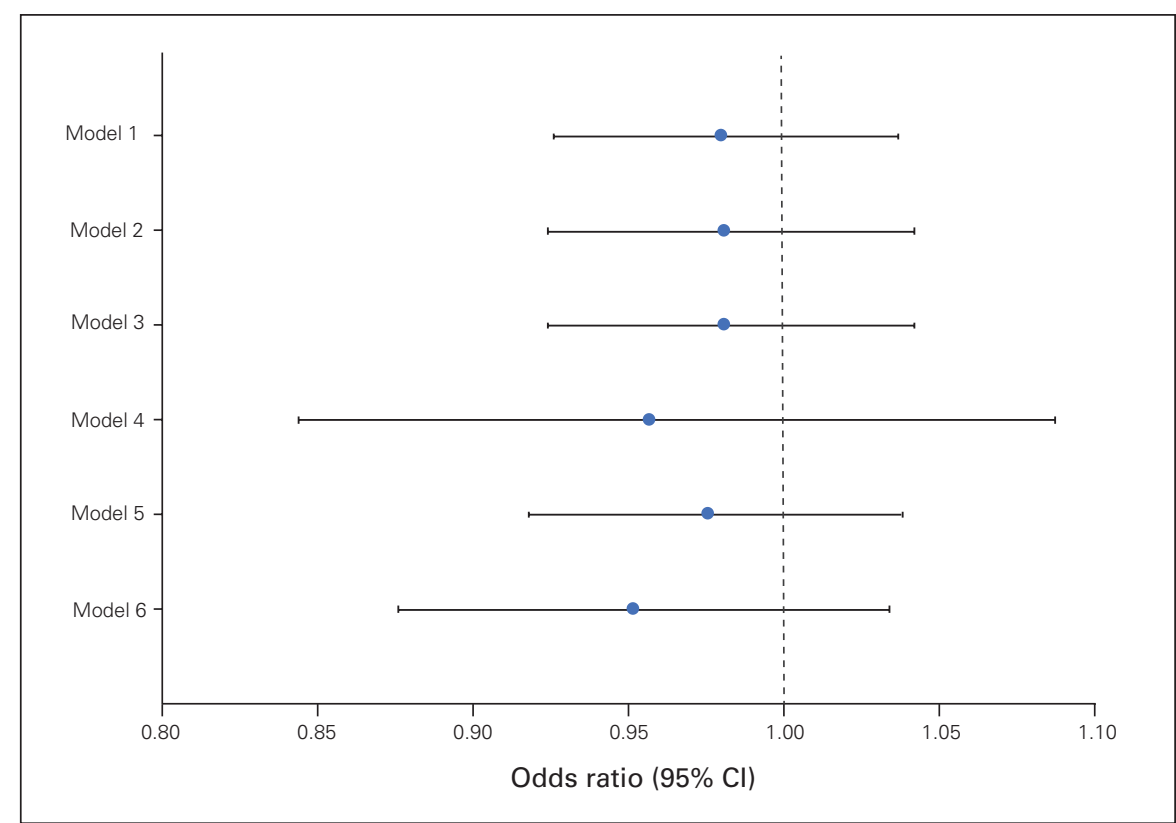

Fig. 1. Associations between provider volume and risk of 30-day postoperative mortality following colorectal surgical interventions in New Brunswick, 2007-2013. Odds ratios were computed per increment of 10 interventions per year, annualized over the preceding 2 years. All models were stratified by sex and by 5 -year age groups. Model 1 : adjusted for year of the intervention, patient comorbidities, admission category, cancer stage, size of patient's home community, and neighbourhood income quintile ( $n$ for deaths $=120$ ). Model 2: same as model 1, plus additional adjustment for provider years of experience, sex and specialization ( $n$ for deaths $=120$ ). Model 3: same as model 2, plus additional adjustment for a categorical indicator of hospital volume ( $n$ for deaths $=$ 120 ). Model 4: same as model 3 , but includes only patients with cancer ( $n$ for deaths $=$ 45). Model 5: same as model 3, but excludes interventions performed by providers who performed fewer than 2 interventions during the study period ( $n$ for deaths $=120$ ). Model 6: same as model 3, but includes only interventions performed by general surgeons ( $n$ for deaths $=80$ ). $\mathrm{Cl}=$ confidence interval.

according to the same 6 modelling scenarios. In all cases, we found significantly reduced odds of complication associated with increased provider volumes (ORs ranged from 0.94 to 0.97 per 10 interventions undertaken per year, annualized over the previous 2 years). For example, in our fully adjusted model (model 3) the OR was 0.94 (95\% CI 0.91-0.96). Results from our sensitivity models show that the association was robust to the various cohort and provider inclusion criteria examined here.

Lastly, we fit models that included an interaction between provider volume and an indicator for colon versus rectal procedures. Approximately $67.7 \%$ of the 9170 procedures were colon related $(n=6210)$ with the remaining $32.3 \%$ rectum related $(n=2960)$. We found no significant evidence of provider volume being associated differentially with colon versus rectal procedures (results not shown).

\section{Discussion}

In this population-based longitudinal cohort study we report reduced odds of complications following colorectal surgery associated with increased provider volumes over the previous 2 years. We found no significant associations between provider volume and postoperative mortality in any of our models. These findings were robust to several restrictions to the cohort and to providers included in the models. This study is among only a handful of Canadian studies to examine this topic, and to our knowledge, it is the first to consider associations between provider volumes and these adverse outcomes following these interventions.

Our study design and methods were generally similar to those of other international studies that have considered associations between volume and health outcomes. The use of 30-day all-cause mortality as an outcome is consistent with a large body of literature; however, our use of these selected complications is novel. Our finding of no association between provider volume and 30-day mortality following colorectal surgery is consistent with the only other Canadian study that considered this outcome with hospital volume. ${ }^{13} \mathrm{~A}$ separate Canadian study, however, found that 5-year survival was associated with increased volume among providers performing rectal cancer resections. ${ }^{15}$ A major challenge for comparing and generalizing findings on this topic and through this literature is the wide heterogeneity in study design, in particular the varying definitions of caseload volume and cohort inclusion criteria. For example, in the existing literature, some studies consider only patients with cancer, some consider only selected interventions and others report results separately for patients who have rectal cancer and patients who have colon cancer. Additionally, categories of specialization and definitions of colorectal specialist vary between jurisdictions. ${ }^{6}$ As noted earlier, most colorectal surgical procedures in New Brunswick are performed by general surgeons. We expect that most, if not all, of the cases included in this study would be broadly representative of those occurring in other provinces. People living in New Brunswick have access to universal health care, the same as those living in other provinces, and the procedures examined here were coded from CCI and are therefore performed similarly elsewhere in the country.

As noted above, somewhat unexpectedly we found that patients whose provider had more years' experience had increased odds of complication. Furthermore, we also found that undergoing one of these procedures in a larger, relatively higher volume hospital was also associated with 
increased odds of complication, after controlling for provider volume and all other variables in our fully adjusted models. In both cases, we expect that these results are due in part to differences in patient case mixes. Specifically, patients in New Brunswick requiring more complex surgical interventions, and who are thus at greatest risk for complications, typically are transferred to the higher volume hospitals and treated by providers with more training and experience. Such information is not captured in the administrative data records available to us and therefore this is something that we cannot demonstrate empirically here.

The findings from this study showed consistently (across several model variations and restrictions) an approximately $6 \%$ reduced odds of a patient experiencing 1 of several common complications per increase in 10 procedures performed annually by the provider, following colorectal surgical procedures in NB. Typical volumes for these procedures among providers in NB were approximately 18 per year. Our analyses also suggest the possibility of reduced likelihood of mortality following colorectal surgery among patients whose procedure was performed by a provider with a higher caseload, although none of our models for mortality showed statistical significance. Our findings also suggest that increased provider volume had similar magnitudes of associations with adverse outcomes among patients for both colon and rectal procedures. Although provider volumes appeared to have a statistically significant effect on the likelihood of adverse outcomes, the size of effect was small, and other factors (i.e., personal characteristics) had larger effects. Nonetheless, these findings suggest that to minimize risk of adverse outcomes, when possible, these interventions should be performed by providers who are actively and regularly performing these procedures.

A key strength of this study is the population-based nature of our cohort, namely that we were able to include all admissions for the interventions of interest in the province over the 7-year period. Additionally, our use of a timevarying definition of volume allowed us to capture changes in provider caseload volumes throughout the follow-up period. Some features of our study that are especially novel include adjusting our models for provider characteristics (e.g., years of experience and specialization) and for both provider and hospital caseload volumes simultaneously.

\section{Limitations}

A limitation of any study based on health administrative data is the lack of personal information on patients that may also be relevant to overall health status, including personal indicators of socioeconomic status, marital status or immigrant status. A limitation of our study design is that our estimates of volume and years of experience do not capture career interruptions (e.g., parental leave) or work undertaken outside of NB. Therefore, in some cases, we may have underestimated actual provider caseloads and overestimated years of experience (i.e., by counting all years since graduation). Unfortunately, data limitations, including small sample sizes and incomplete information on some surgical details, prevented us from considering results separately among patients admitted to the lower and higher volume hospitals, and according to provider sex or specialty. For example, our data included only the primary specialty of each provider. This lack of information limited our ability to evaluate the importance of specialization or subspecialty training, which has been shown elsewhere to be associated with disease recurrence and 
long-term survival following rectal cancer surgery. ${ }^{15}$ Similarly, there are other dimensions of surgical outcomes that we were unable to examine (e.g., laparoscopic v. open resection), but on the basis of sample sizes, previous work and practicality, we believe that we were able to include the most relevant ones.

\section{Conclusion}

The findings of this study corroborate those from the broader literature, which suggest generally that increased provider experience is associated with better postoperative outcomes. Future studies on this topic may wish to explore other indicators of provider experience or measures of caseload volume, including shorter or longer term estimates of volume, outcomes beyond 30 days and other outcomes, such as length of hospital stay or patient-reported outcomes following colorectal interventions. Given the data sets and methods used in this analysis, our results should be generalizable to other Canadian provinces.

Affiliations: From the Department of Sociology, University of New Brunswick, Fredericton, N.B. (Crouse); the New Brunswick Institute for Research, Data and Training, University of New Brunswick, Fredericton, N.B. (Crouse, Boudreau, Leonard, McDonald); the Department of Economics, University of New Brunswick, Fredericton, N.B. (Leonard, McDonald); and the Faculty of Medicine, Dalhousie University, Halifax, N.S. (Pawluk).

Funding: This study was supported by the Maritime SPOR SUPPORT Unit (MSSU), which receives financial support from the Canadian Institutes of Health Research, the Nova Scotia Department of Health and Wellness, the New Brunswick Department of Health, the Nova Scotia Health Research Foundation and the New Brunswick Health Research Foundation. The opinions, results and conclusions reported in this paper are those of the authors and are independent from the funding sources. No endorsement by the MSSU or the named funding partners is intended or should be inferred. This work was also supported by the New Brunswick Department of Health under a data-sharing agreement with the New Brunswick Institute for Research, Data and Training at the University of New Brunswick. The results and conclusions are those of the authors and no official endorsement by the Government of New Brunswick was intended or should be inferred.

Competing interests: None declared.

Contributors: All authors conceived the study. J. Boudreau and J. McDonald acquired the data, which D. Crouse, J. Boudreau, P. Leonard and J. McDonald analyzed. D. Crouse, J. Boudreau, P. Leonard and J. McDonald drafted the manuscript, which J. Boudreau, P. Leonard, K. Pawluk and J. McDonald critically revised. All authors gave final approval of the version to be published.

\section{References}

1. Birkmeyer JD, Stukel TA, Siewers AE, et al. Surgeon volume and operative mortality in the United States. NEngl F Med 2003;349:2117-27.

2. Birkmeyer JD, Siewers A, Finlayson E, et al. Hospital volume and surgical mortality in the United States. N Engl f Med 2002;346:1128-37.

3. Morche J, Mathes T, Pieper D. Relationship between surgeon volume and outcomes: a systematic review of systematic reviews. Syst Rev 2016;5:204.

4. Pieper D, Mathes T, Neugebauer E, et al. State of evidence on the relationship between high-volume hospitals and outcomes in surgery: a systematic review of systematic reviews. 7 Am Coll Surg 2013;216: 1015-1025.e18.

5. Archampong D, Borowski DW, Dickinson HO. Impact of surgeon volume on outcomes of rectal cancer surgery: a systematic review and meta-analysis. Surgeon 2010;8:341-52.

6. Archampong D, Borowski D, Wille-Jørgensen P, et al. Workload and surgeon's specialty for outcome after colorectal cancer surgery. Cocbrane Database Syst Rev 2012;14:CD005391.

7. Iversen LH, Harling H, Laurberg S, et al. Influence of caseload and surgical speciality on outcome following surgery for colorectal cancer: a review of evidence. Part 2: long-term outcome. Colorectal Dis 2007;9:38-46.

8. Salz T, Sandler RS. The effect of hospital and surgeon volume on outcomes for rectal cancer surgery. Clin Gastroenterol Hepatol 2008;6:1185-93.

9. Huo YR, Phan K, Morris DL, et al. Systematic review and a metaanalysis of hospital and surgeon volume/outcome relationships in colorectal cancer surgery. 7 Gastrointest Oncol 2017;8:534-46.

10. Rogers SO, Wolf RE, Zaslavsky AM, et al. Relation of surgeon and hospital volume to processes and outcomes of colorectal cancer surgery. Ann Surg 2006;244:1003-11.

11. Simunovic M, To T, Baxter N, et al. Hospital procedure volume and teaching status do not influence treatment and outcome measures of rectal cancer surgery in a large general population. 7 Gastrointest Surg 2000;4:324-30.

12. Simunovic M, Rempel E, Thériault ME, et al. Influence of hospital characteristics on operative death and survival of patients after major cancer surgery in Ontario. Can 7 Surg 2006;49:251-8.

13. Urbach DR, Baxter NN. Does it matter what a hospital is 'high volume' for? Specificity of hospital volume-outcome associations for surgical procedures: analysis of administrative data. Qual Saf Health Care 2004;13:379-83.

14. McColl RJ, McGahan CE, Cai E, et al. Impact of hospital volume on quality indicators for rectal cancer surgery in British Columbia, Canada. Am 7 Surg 2017;213:388-94.

15. Porter GA, Soskolne CL, Yakimets WW, et al. Surgeon-related factors and outcome in rectal cancer. Ann Surg 1998;227:157-67.

16. Burns EM, Bottle A, Almoudaris AM, et al. Hierarchical multilevel analysis of increased caseload volume and postoperative outcome after elective colorectal surgery. Br 7 Surg 2013;100:1531-8.

17. Burns EM, Mamidanna R, Currie A, et al. The role of caseload in determining outcome following laparoscopic colorectal cancer resection: an observational study. Surg Endosc 2014;28:134-42.

18. Fischer C, Lingsma HF, Van Leersum N, et al. Comparing colon cancer outcomes: the impact of low hospital case volume and casemix adjustment. Eur 7 Surg Oncol 2015;41:1045-53.

19. Nikolian VC, Kamdar NS, Regenbogen SE, et al. Anastomotic leak after colorectal resection: a population-based study of risk factors and hospital variation. Surgery 2017;161:1619-27.

20. Statistics Canada. Postal code conversion file plus $(P C C F+)$ version $6 D$, reference guide. Cat no. 82-F0086-XDB. Ottawa: Statistics Canada; 2016.

21. Healy MA, Gilliland JA. Quantifying the magnitude of environmental exposure misclassification when using imprecise address proxies in public health research. Spat Spatiotemporal Epidemiol 2012;3:55-67.

22. Khan S, Pinault L, Tjepkema M, et al. Positional accuracy of geocoding from residential postal codes versus full street addresses. Health Rep 2018;29:3-9.

23. Crouse DL, Leonard PSJ, Boudreau J, et al. Associations between provider and hospital volumes and postoperative mortality following total hip arthroplasty in New Brunswick: results from a provinciallevel cohort study. Can 7 Surg 2018;61:88-93.

24. Canadian Institute for Health Information. Indicator library: peer group methodology. Ottawa: Canadian Institute for Health Information; 2016. 